\title{
Cognitive function in Prefrail and frail community-dwelling older adults in China
}

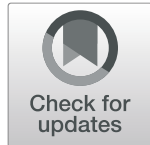

Lina Ma ${ }^{1,2}$, Li Zhang ${ }^{1}$, Fei Sun ${ }^{2}$, Yun Li ${ }^{1}$ and Zhe Tang ${ }^{2 *}$

\begin{abstract}
Background: Physical frailty, characterized by reduced physiologic complexity and ability to cope with stressors, is closely associated with cognitive impairment, which increases the risk of poor clinical outcomes. To better capture the association between frailty and cognitive impairment, a new construct, cognitive frailty, has been proposed. Cognitive frailty is a clinical condition characterized by the simultaneous presence of physical frailty and cognitive impairment. There is little evidence on the relationship between physical frailty and cognition, as well as cognitive frailty, in Chinese older adults. We aimed to elucidate whether physical frailty is associated with cognitive impairment in an older Chinese population.

Methods: Data were obtained from the China Comprehensive Geriatric Assessment Study. The sample comprised 3202 community-dwelling adults, aged 60 years and older, from seven Chinese cities. Physical frailty was assessed using a modified, four-item version of the Fried criteria, according to frailty phenotype. Cognitive function was assessed using the Mini-Mental State Examination (MMSE).

Results: The prevalence of physical frailty, prefrailty, cognitive impairment, and cognitive frailty was 9.9, 33.9, 7.5, and $2.3 \%$, respectively (weighted: $8.8,33.8,6.5$, and $2.0 \%$ ). The prevalence of the combination of prefrail/frail and cognitive impairment was $5.1 \%$ (weighted $4.5 \%$ ). Frail participants performed worse on global cognition and all cognitive domains than robust and prefrail participants. The MMSE total score was positively correlated with walking speed and negatively correlated with age and frailty. A multivariate logistic regression revealed that after adjusting for age, gender, education level, living area, and chronic diseases, frailty, exhaustion, slowness, and inactivity were significantly associated with poor global cognition.

Conclusions: The standard prevalence of physical frailty, prefrailty, cognitive impairment, and cognitive frailty in community-dwelling older adults in China was 8.8, 33.8, 6.5, and 2.0\%, respectively. Frailty, exhaustion, slowness, and inactivity were significantly associated with poor global cognition.
\end{abstract}

Keywords: Frailty, Cognition, Cognitive frailty, Older adults

\section{Background}

Aging is associated with both physical and cognitive decline. Frailty is a medical syndrome characterized by diminished physical function and reduced age-related physiologic reserve leading to decreased resistance to stressors and increased vulnerability to disability, morbidity, and mortality $[1,2]$. Therefore, frailty has become

\footnotetext{
* Correspondence: tangzhe@sina.com

${ }^{2}$ Beijing Geriatric Healthcare Center, Xuanwu Hospital, Capital Medical University, Beijing Institute of Geriatrics, Key Laboratory on

Neurodegenerative Disease of Ministry of Education, Beijing Institute for

Brain Disorders, China National Clinical Research Center for Geriatric

Disorders, Beijing 100053, China

Full list of author information is available at the end of the article
}

a major public health issue. Since frailty better captures variations in health risks than chronological age [3], it has been suggested as a better predictor of health and well-being and might be a better indicator of adverse outcomes among older adults [4]. However, despite its wide use in clinical practice, there is no consensus on the definition of frailty. The most common approach to measure frailty is the Fried frailty phenotype with biological underpinnings encompassing five components including unintentional weight loss, exhaustion, weakness, slowness, and inactivity [1]. Another important measurement is Rockwood's frailty index, which considers frailty in terms of deficit accumulation [5, 6]. The

(c) The Author(s). 2019 Open Access This article is distributed under the terms of the Creative Commons Attribution 4.0 International License (http://creativecommons.org/licenses/by/4.0/), which permits unrestricted use, distribution, and 
close association of frailty and cognitive impairment (CI) increases the risk of mortality in later life. It is estimated that 115 million people worldwide will have dementia by 2050. Declined cognitive reserve leads to mild cognitive impairment (MCI) and dementia, which runs parallel to the course of physical frailty. CI is associated with increased risk of walking speed decline and future frailty $[7,8]$, whereas frailty predicts cognitive decline/incident dementia [9, 10]. Lee et al. found that the association between frailty and mortality was moderated by baseline cognitive status [11]. As early detection and intervention development to prevent poor clinical outcomes are becoming increasingly important, taking cognition into account may allow for better prediction of adverse outcomes of frailty in later life.

Although a growing number of studies are now focusing on the relationship between frailty and CI, literature has generally considered them as two different entities. Given the many risk factors and underlying mechanisms common to physical frailty and CI [12], the International Academy on Nutrition and Aging and the International Association of Gerontology and Geriatrics have proposed a new construct, "cognitive frailty" (CF) - a clinical condition that describes the simultaneous presence of physical frailty and MCI [13]-providing a framework for research to identify individuals with CI caused by non-neurodegenerative conditions. Although there is no universal consensus regarding $\mathrm{CF}$, it has been used in many recent studies, where it has been associated with greater risk of adverse outcomes [12, 14]. With aging, older persons on a CF trajectory exhibit the greatest burden of nursing home admission and disability [15].

So far, there is still little evidence on the relationship between physical frailty and cognition in Chinese older adults. We previously reported a $3.3 \%$ prevalence of $\mathrm{CF}$ in mainland China with the definition of CF defined by frailty index and the Mini-Mental State Examination (MMSE) and identified its related factors [16]; however, whether physical frailty was associated with worse cognition is still unknown. Thus, we conducted this study to further elucidate whether physical frailty is associated with $\mathrm{CI}$ in an older Chinese population.

\section{Methods}

\section{Study design}

Data were obtained from the China Comprehensive Geriatric Assessment Study (CCGAS), and stratified, multiple-stage, random, and cluster sampling methods were used to recruit community-dwelling participants in China aged 60 years and older between 2011 and 2012 . In the first stage, three cities (Beijing, Xi'an, and Harbin) were chosen from the northern cities, and four cities (Chengdu, Chongqing, Changsha, and Shanghai) were chosen from the southern cities. These selected seven cities represented the six main regions of China $[17,18]$. In the second stage, older adults residing in different urban and rural areas were selected, using age group and sex ratios based on information about the population composition from the Sixth National Census (2010). Of the 6867 community-dwelling older adults, 3202 individuals without a history of dementia and with a physical frailty assessment and MMSE data were included. Compared to the 3202 included participants, those excluded were older $(71.94 \pm 8.03$ vs. $70.14 \pm$ 7.08 years, $p<0.001)$, had worse cognitive function (MMSE score: $25.85 \pm 5.22$ vs. $26.71 \pm 4.69, p<0.001$ ), were more disabled $(11.9 \%$ vs. $4.2 \%, p<0.001)$, and had more chronic diseases ( $\geq 2: 64.4 \%$ vs. $53.9 \%, p<$ $0.001)$.

\section{Data collection}

The clinical and demographic variables related to each subject were collected using a questionnaire administered in a face-to-face interview by trained staff, along with a physical examination. The physical examination was conducted at home or at a central location. Data gathered included the sociodemographic characteristics (e.g., gender, age, education level, marital status, income), anthropometric measurements (e.g., height, weight), health status, personal habits, mental health (e.g., cognition, depression) and variables regarding frailty assessment were also collected. Waist-to-hip ratio (WHR) was calculated as waist measurement divided by hip measurement. Body mass index (BMI) was calculated by dividing the weight in kilogram by height in meter squared and BMI cutoffs were based on Asian adjustments. Income was defined as the amount of money received monthly from an employer or an individual's from family (for those without a job). Participants were considered to have a medical condition if they had a self-reported history of chronic disease diagnosed by a doctor. Functional ability was assessed based on the capacity of individuals to perform activities of daily living (ADL) and instrumental activities of daily living (IADL). The list of activities consists of 14 items (Eating, grooming, dressing, transferring in and out of bed, bathing, walking inside the house, using the toilet, cooking, managing finances, driving or using public transportation, shopping, walking $250 \mathrm{~m}$, cutting toenails, and climbing stairs), and an individual's performance on each item is classified as independent, partially dependent and completely dependent, scored 1, 2, and 3, respectively.

\section{Cognitive assessment}

Cognitive function was assessed using a 30-question MMSE; each correctly answered question was awarded one point, whereas incorrect or no answers were awarded zero points. The total score ranged from 0 to 
30 points. A recent study in a Chinese population showed age-, gender-, education-, and residence-specific reference norms for the MMSE [19]. The thresholds for those who were illiterate, or attended at most primary school, middle school, or university were $\leq 17$, $\leq 20$, $\leq$ 22 , and $\leq 24$, respectively. Participants who scored below the threshold value for their education group were classified as CI [20]. MMSE assesses ten different cognitive domains: comprehension (range 0-3), reading (range 01 ), naming (range 0-2), drawing (range 0-1), writing (range 0-1), repetition/registration (range $0-3$ ), orientation to time (range $0-5$ ), orientation to place (range $0-5$ ), recall (range 0-3) and attention (range 0-6).

\section{Frailty assessment tool}

Physical frailty was assessed using the modified, four-item version of the Fried criteria (weakness was not considered), according to the frailty phenotype derived from the Cardiovascular Health Study. The four items are unintentional weight loss, self-rated exhaustion, slow walking speed, and inactivity. Weight loss was defined as BMI less than $18.5 \mathrm{~kg} / \mathrm{m}^{2}$. Exhaustion was indicated by a self-response as "yes" to "Is it hard for you to start on new projects" and "no" to "Do you feel full of energy" from the Geriatric Depression Scale. Inactivity was defined as exercising for $<3 \mathrm{~h} /$ week over the past 12 months. Walking speed was evaluated with a $20-\mathrm{m}$ walking test. Slowness was defined as the lowest quintile of the walking speed, adjusted for gender and standing height of the participants: $<0.67 \mathrm{~m} / \mathrm{s}$ for males with height $>166 \mathrm{~cm},<0.65 \mathrm{~m} / \mathrm{s}$ for males with height $\leq 166$ $\mathrm{cm},<0.63 \mathrm{~m} / \mathrm{s}$ for females with height $>155 \mathrm{~cm}$, and $<$ $0.57 \mathrm{~m} / \mathrm{s}$ for females with height $\leq 155 \mathrm{~cm}$. The fulfillment of two or more of the above criteria on the frailty scale was classified as frail, the fulfillment of one was considered prefrail, and no criterion was nonfrail.

\section{CF criteria}

Participants positive for both Fried frailty phenotype and cognitive assessment were classified as having CF.

\section{Statistical analysis}

All statistical analyses were performed using the SPSS for Windows Version 11.5 (SPSS Inc., Chicago, IL). The chi-squared test was used to compare categorical data, which were expressed as numbers and percentages. Continuous data were analyzed using 1-way analysis of variance and were expressed as means and standard deviations. Spearman's rank correlation coefficient was used to determine correlations. The standard rates calculated using the national standard population composition ratio as at the Sixth National Census (2010). A forward stepwise logistic regression was conducted to explore the association between the related factors as independent variables and $\mathrm{CI}$ as the dependent variable. Hypothesis testing was two-sided, using a level of significance of 0.05 .

\section{Results}

According to the Fried criteria, of the 3202 older adults without dementia, 317, 1087, and 1798 participants were frail, prefrail, and robust and the prevalence was 9.9, 33.9, and 56.2\% (weighted: 8.8, 33.8, and 57.4\%), respectively. A total of 241 participants (prevalence $7.5 \%$, weighted: $6.5 \%$ ) were cognitively impaired. The prevalence of CF was $2.3 \%$ (weighted 2.0\%). A total of 164 participants were both prefrail/frail and CI (5.1\%, weighted $4.5 \%$ ). Compared to robust adults, both frail and prefrail older adults were older, had lower BMI, slower walking speed, and performed worse on ADL and IADL. Older adults living in rural areas or those with low monthly incomes had a higher prevalence of frailty (Table 1).

Table 2 shows the effect of frailty on cognition. Frail participants performed worse on global cognition and all the ten domains than both robust and prefrail participants. Prefrail residents scored statistically less in the areas of global cognition, reading, drawing, writing, repetition, orientation to time, orientation to place, recall, and attention than robust older adults. We further conducted correlation between the MMSE score and many variables; the MMSE score was positively correlated with walking speed $(r=0.244, p<0.001)$ and negatively correlated with age $(r=-0.2426, p<0.001)$, and frailty phenotype score $(r=-0.2835, p<0.001)$ (Fig. 1$)$.

A multivariate logistic regression revealed that after adjusting for age, gender, education level, living area, and chronic diseases, frailty [hazard ratio (HR): 2.571(1.7893.695), $p<0.001$ ], exhaustion [HR: 2.099(1.389-3.172), $p<$ 0.001 ], slowness [HR: 1.859(1.327-2.606), $p<0.001$ ], and inactivity [HR: 1.709(1.250-2.335), $p=0.001$ ] were significantly associated with global cognition. Weight loss was not independently associated with cognition (Table 3).

\section{Discussion}

In the nationwide survey, we found the standard overall prevalence of physical frailty, prefrailty, CI, and CF in Chinese older adults to be $8.8,33.8,6.5$, and $2.0 \%$, respectively, which was in accordance with previous studies $[18,21]$. Recent studies have shown the prevalence of physical frailty, CI, and CF to be 5.1, 5.5, and $1.1 \%$, respectively, in Japanese older adults [22], and the prevalence of CF was $4.4 \%$ in Italian older adults [23]. A recent review estimated the prevalence of $\mathrm{CF}$ to be as low as $1.0-1.8 \%$ in community settings [24], where it is associated with a high risk of disability, dementia, poor quality of life, and death [22-24]. The aforementioned studies suggest that CF might be a useful concept for 
Table 1 Characteristics of robust, prefrail and frail participants

\begin{tabular}{llll}
\hline & Robust & Prefrail & Frail \\
\hline Number (\%) & $1798(56.2)$ & $1087(33.9)$ & $317(9.9)$ \\
Age (years) & $69.25 \pm 6.71$ & $70.36 \pm 7.00^{*}$ & $74.39 \pm 7.83^{*} \Delta$ \\
Gender (male, \%) & $798(44.4)$ & $468(43.1)$ & $140(44.2)$ \\
Area (rural, \%) & $551(30.6)$ & $345(31.7)$ & $154(48.6)^{*} \Delta$ \\
Income (yuan)/month & $2463.04 \pm 1864.63$ & $2303.35 \pm 1576.80$ & $1829.18 \pm 1710.89^{*} \Delta$ \\
BMI (kg/m²) & $24.31 \pm 3.16$ & $23.66 \pm 3.56^{*}$ & $22.67 \pm 3.98^{*} \Delta$ \\
WHR & $0.8720 \pm 0.0636$ & $0.8724 \pm 0.0585$ & $0.8718 \pm 0.0695$ \\
Walking speed (m/s) & $1.01 \pm 0.32$ & $0.77 \pm 0.34^{*}$ & $0.60 \pm 0.21^{*} \Delta$ \\
ADL & $7.01 \pm 0.10$ & $7.05 \pm 0.42^{*}$ & $7.38 \pm 1.15^{*} \Delta$ \\
IADL & $7.15 \pm 0.78$ & $7.34 \pm 1.27^{*}$ & $8.76 \pm 2.97^{*} \Delta$ \\
\hline
\end{tabular}

Abbreviations: $B M I$ body mass index, WHR waist hip ratio, $A D L$ activities of daily living, IADL instrumental activities of daily living

${ }^{*} P<0.05$, compared to robust group; ${ }^{\Delta} P<0.05$, compared to prefrail group

clinicians and a potentially preventive or therapeutic target for dementia and disability in later life.

We found that frail participants had worse global cognition in all the domains of the MMSE than both robust and prefrail participants, and prefrail residents scored statistically lower in the areas of global cognition, reading, drawing, writing, repetition, orientation to time, orientation to place, recall, and attention than robust older adults. Other studies also found worse cognitive functioning in frail patients, controlling for age and gender [23]; furthermore, robust but cognitively impaired participants are more likely to be prefrail/frail than normal cognitive participants [25], which indicates that CI might take part in the pathogenic mechanisms of frailty. Frailty was also associated with metacognitive executive dysfunction [26]. There is a cumulative but no interactive effect of frailty and $\mathrm{CI}$ in the prediction for mortality [27].

We further found that the MMSE total score was positively correlated with walking speed and negatively correlated with age and frailty total score. A multivariate logistic regression revealed that after adjusting for age, gender, education level, living area, and chronic diseases, global cognition was significantly lower in participants with frailty, exhaustion, slowness, and inactivity. A previous study had found that an increase in age is accompanied by a cognitive decline with decreased grip strength, slower walking speed, and more severe depression [28]. A recent review revealed that physical activity was associated with changes in executive function and word recall and global cognitive function was associated with grip strength, walking speed, and exhaustion [29]. Physical frailty is a stronger indicator of everyday and global cognition than age [30]; both baseline status and within-person changes in frailty were predictive of cognitive trajectories [10]. The information process speed and decline rate over time may have an important role in the onset of frailty [31]. Slow walking speed was associated with CI [32]. After controlling for other variables, each 0.10 increase in baseline frailty was associated with a 0.01 increase in $\mathrm{CI}$ at follow-up,

Table 2 The effect of frailty on cognition

\begin{tabular}{llll}
\hline & Robust $(n=1798)$ & Prefrail $(n=1087)$ & Frail $(n=317)$ \\
\hline MMSE total score & $27.54 \pm 3.72$ & $26.46 \pm 4.86^{*}$ & $22.79 \pm 6.59^{*} \Delta$ \\
Comprehension & $2.92 \pm 0.32$ & $2.87 \pm .045$ & $2.70 \pm 0.67^{*} \Delta$ \\
Reading & $0.96 \pm 0.19$ & $0.94 \pm 0.23^{*}$ & $0.84 \pm 0.37^{*} \Delta$ \\
Naming & $1.99 \pm 0.13$ & $1.99 \pm 0.14$ & $1.95 \pm 0.30^{*} \Delta$ \\
Drawing & $0.74 \pm 0.44$ & $0.69 \pm 0.46^{*}$ & $0.48 \pm 0.50^{*} \Delta$ \\
Writing & $0.97 \pm 0.20$ & $0.94 \pm 0.24^{*}$ & $0.84 \pm 0.37^{*} \Delta$ \\
Repetition/registration & $2.90 \pm 0.40$ & $2.80 \pm 0.51^{*}$ & $2.61 \pm 0.79^{*} \Delta$ \\
Orientation to time & $4.66 \pm 0.89$ & $4.46 \pm 1.15^{*}$ & $3.66 \pm 1.67^{*} \Delta$ \\
Orientation to place & $4.80 \pm 0.66$ & $4.69 \pm 0.85^{*}$ & $4.20 .291 \pm 1.35^{*} \Delta$ \\
Recall & $2.52 \pm 0.79$ & $2.39 \pm 0.89^{*}$ & $1.93 \pm 1.10^{*} \Delta$ \\
Attention & $5.09 \pm 1.59$ & $4.69 \pm 1.85^{*}$ & $3.58 \pm 2.19^{*} \Delta$ \\
\hline
\end{tabular}

${ }^{*} P<0.05$, compared to robust group; ${ }^{\Delta} P<0.05$, compared to prefrail group 

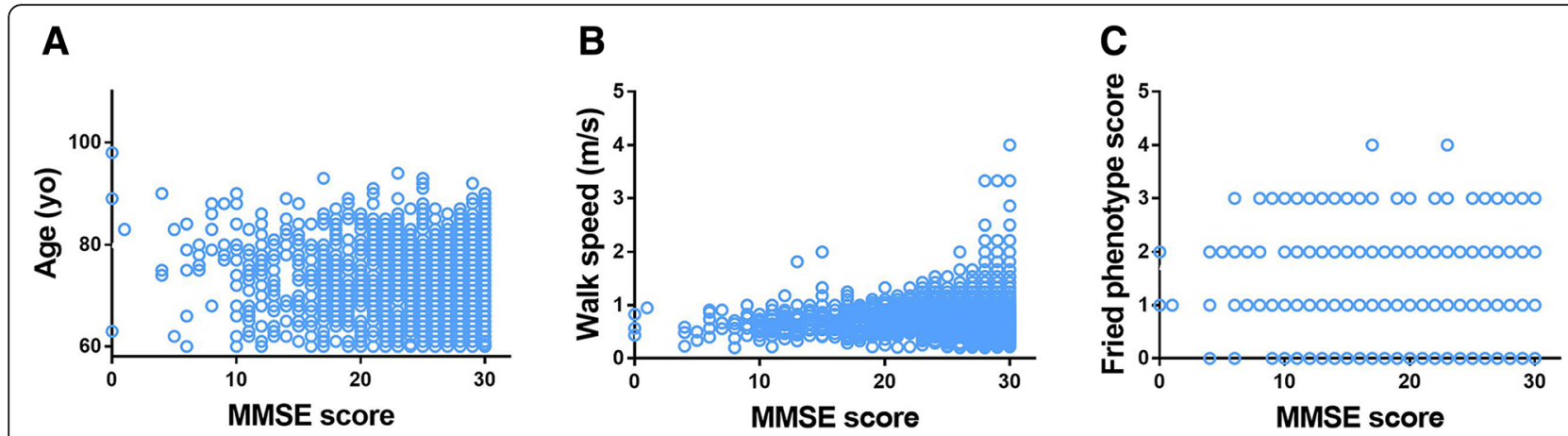

Fig. 1 Correlation between MMSE total score with age and frailty. Spearman's rank correlation test was used to determine the relationships between MMSE total score with age (a), walking speed (b), and Fried phenotype score (c) among adults aged 60 years and older in China by CCGAS, 2011-2012. The total sample population in the analysis was 3202

while each 0.1 increase in baseline CI was associated with a 0.003 increase in frailty at follow-up [33]. In another study, although frailty was associated with poor baseline cognitive performance, there were no effects on slopes of cognition, suggesting that frailty was not associated with cognitive decline, which might be explained by the fact that frailty-related cognitive deficits may exist independent of mechanisms underpinning neurodegenerative disorders [34], providing further evidence of the importance of the notion of CF.

Given that early-stage intervention may be more effective in older adults and that the frailty continuum is reversible [35], prefrailty, an intermediate and preclinical state, should be included in the concept of CF. We found that the prevalence of the combination of prefrail/ frail and CI was 5.1\% (weighted 4.5\%). Another study found that prefrailty combined with lower cognition scores at baseline was associated with higher risks of poor quality of life, incident physical limitation, increased cumulative hospital stay, and mortality [25]. Prefrailty was associated with worse memory and processing speed [36]. Exercises may delay or reverse cognitive decline, indicating that early detection of $\mathrm{CF}$ has public health implications [37].

To date, there is no consensus on the definition of CF or on how CI and physical frailty should be measured. Numerous frailty tools make an operational definition of $\mathrm{CF}$ and the development of preventive intervention strategies more difficult. For example, a study found that the prevalence of frailty was $7.5 \%$ using the Edmonton Frail Scale but 30\% using CHS criteria indicating that the identification of frailty in $\mathrm{CI}$ is partly dependent on assessment methods [38]. A systematic review identified seven methods of cognitive assessment in frailty operationalization such as dementia as comorbidity and objective cognitive screening instruments [39]. Delayed recall, language, and praxis used as criteria for $\mathrm{CI}$ in combination of prefrailty can predict adverse outcomes [25]. Very recently, $\mathrm{Yu}$ et al. found that the use of a single cognitive domain may be effective in characterizing CI groups, and that the use of prefrailty also identifies a subset of individuals at risk of progressing to frailty [25]. In this study, we chose the Fried phenotype for frailty assessment and the MMSE for cognition measurement, which were easy to use in clinical work and for large

Table 3 Multivariate logistic regression of frailty components on global cognition

\begin{tabular}{|c|c|c|c|c|c|c|}
\hline \multirow{2}{*}{$\begin{array}{l}\text { Frailty } \\
\text { component }\end{array}$} & \multicolumn{2}{|l|}{ Model 1} & \multicolumn{2}{|l|}{ Model 2} & \multicolumn{2}{|l|}{ Model 3} \\
\hline & $\mathrm{HR}(95 \% \mathrm{Cl})$ & $P$ & $\mathrm{HR}(95 \% \mathrm{Cl})$ & $\mathrm{P}$ & $\mathrm{HR}(95 \% \mathrm{Cl})$ & $P$ \\
\hline Frailty & $5.076(3.750-6.871)$ & $<0.001$ & $4.270(3.117-5.851)$ & $<0.001$ & $2.571(1.789-3.695)$ & $<0.001$ \\
\hline Weight loss & $1.942(1.176-3.206)$ & 0.010 & $1.695(1.016-2.827)$ & 0.043 & - & 0.103 \\
\hline Exhaustion & $3.346(2.392-4.680)$ & $<0.001$ & $2.759(1.953-3.899)$ & $<0.001$ & $2.099(1.389-3.172)$ & $<0.001$ \\
\hline Slowness & $2.406(1.821-3.179)$ & $<0.001$ & $2.081(1.562-2.771)$ & $<0.001$ & $1.859(1.327-2.606)$ & $<0.001$ \\
\hline Inactivity & $2.762(2.105-3.623)$ & $<0.001$ & $2.654(2.013-3.499)$ & $<0.001$ & $1.709(1.250-2.335)$ & 0.001 \\
\hline
\end{tabular}

Model 1: not adjusted

Model 2: adjusted for age and gender

Model 3: model 2 adjusted for education, living area and chronic diseases 
populations. We further included the prefrailty in the construct of $\mathrm{CF}$, which provided the evidence that prefrailty in the CF in Chinese older adults.

The present study has several limitations. First, the cross-sectional design made it difficult to interpret the cause-effect relationship of the association between physical frailty and CI. Second, we used a four-item version of the Fried criteria, as weakness (measured by grip strength) was not considered in the survey and weight loss was defined as low BMI, rather than as a quantified change in weight over time. Third, cognition was assessed by the MMSE instead of a battery of neuropsychological measurements. Brain imaging data were lacking in the current study. However, our study also has several strengths. The CCGAS, a nationwide survey in China, is based on well-established cluster, stratified, and random selection statistical sampling techniques, and the seven cities were representative of the six main regions in China. Furthermore, we provided the first empirical epidemiological evidence of the prevalence of $\mathrm{CF}$ based on the Fried phenotype in mainland China.

\section{Conclusion}

Our study provides epidemiological evidence of the prevalence of physical frailty, prefrailty, CI, and CF in Chinese older adults as well as the association between physical frailty and CI. Frailty and its components except weight loss were independently associated with CI. Combined with the growing epidemiological evidence from other countries, physical frailty is linked to $\mathrm{CI}$ and the concept of $\mathrm{CF}$ provides a new approach to prevent later-life function decline and dementia. However, the mechanisms underlying the relationship between physical frailty and $\mathrm{CI}$, as well as CF should be further studied.

\section{Abbreviations}

ADL: Activities of daily living; BMI: Body mass index; CCGAS: China Comprehensive Geriatric Assessment Study; CF: Cognitive frailty; HR: Hazard ratio; IADL: Instrumental activities of daily living; $\mathrm{MCl}$ : Mild cognitive impairment; MMSE: Mini-Mental State Examination; WHR: Waist-to-hip ration

\section{Acknowledgments}

We acknowledge all the people who participated in the CCGAS from the following hospitals: Xuanwu Hospital, Beijing Hospital, Beijing Institute of Geriatrics, General Hospital of PLA, Peking University Third Hospital, The First Affiliated Hospital of Xi'an Jiaotong University, Xijing Hospital of The Fourth Military Medical University, The First Affiliated Hospital of Harbin Medical University, West China Hospital of Sichuan University, First Affiliated Hospital of Chongqing Medical University, Xiangya Hospital of Central South University, The Third Xiangya Hospital of Central South University, and Ruijin Hospital of Shanghai Jiao Tong University School of Medicine.

\section{Funding}

This work was supported by and the National Natural Science Foundation of China (81600927), the Beijing Municipal Administration of Hospitals Clinical Medicine Development of Special Funding Support (ZYLX201706), the Ministry of Health Welfare Industry Special Fund (201002011), Beijing Municipal Health Bureau Research Fund (Jing 17-12), and Milstein Medical Asian American Partnership Foundation Project Award in Geriatrics (2018).
Availability of data and materials

The datasets used and/or analysed during the current study are available from the corresponding author on reasonable request.

\section{Authors' contributions}

LM and ZT designed the study; LZ, FS and YL analysed and interpreted the data; LM was a major contributor in writing the manuscript. All authors read and approved the final manuscript.

\section{Ethics approval and consent to participate}

This study was approved by the ethics review board of Xuanwu Hospital, Capital Medical University and all the participants provided written informed consent.

\section{Consent for publication}

Not applicable.

\section{Competing interests}

The authors declare that they have no competing interests.

\section{Publisher's Note}

Springer Nature remains neutral with regard to jurisdictional claims in published maps and institutional affiliations.

\section{Author details}

${ }^{1}$ Department of Geriatrics, Xuanwu Hospital, Capital Medical University, Beijing 100053, China. ${ }^{2}$ Beijing Geriatric Healthcare Center, Xuanwu Hospital, Capital Medical University, Beijing Institute of Geriatrics, Key Laboratory on Neurodegenerative Disease of Ministry of Education, Beijing Institute for Brain Disorders, China National Clinical Research Center for Geriatric Disorders, Beijing 100053, China.

Received: 22 May 2018 Accepted: 4 February 2019

Published online: 27 February 2019

\section{References}

1. Fried LP, Tangen CM, Walston J, Newman AB, Hirsch C, Gottdiener J, et al. Frailty in older adults: evidence for a phenotype. J Gerontol Ser A Biol Sci Med Sci. 2001;56:M146-57. https://doi.org/10.1093/gerona/56.3.M146.

2. Ma L, Zhang L, Tang Z, Sun F, Diao L, Wang J, et al. Use of the frailty index in evaluating the prognosis of older people in Beijing: a cohort study with an 8-year follow-up. Arch Gerontol Geriatr. 2016;64:172-7. https://doi.org/10. 1016/j.archger.2015.11.002.

3. Lacas A, Rockwood K. Frailty in primary care: a review of its conceptualization and implications for practice. BMC Med. 2012;10:4. https:// doi.org/10.1186/1741-7015-10-4.

4. Ma L. Current situation of Frailty screening tools for older adults. J Nutr Health Aging. 2018:(1):-8. https://doi.org/10.1007/s12603-018-1123-4.

5. Rockwood K, Song X, MacKnight C. A global clinical measure of fitness and frailty in elderly people. CMAJ. 2005;173:489-495.

6. Rockwood K, Andrew M, Mitnitski A. A comparison of two approaches to measuring frailty in elderly people. J Gerontol A Biol Sci Med Sci. 2007;62: 738-43.

7. Atkinson HH, Rosano C, Simonsick EM, Williamson JD, Davis C, Ambrosius WT, et al. Cognitive function, gait speed decline, and comorbidities: the health, aging and body composition study. J Gerontol A Biol Sci Med Sci. 2007:62:844-50.

8. Feng L, Zin Nyunt MS, Gao Q, Feng L, Yap KB, Ng T-P. Cognitive Frailty and adverse health outcomes: findings from the Singapore longitudinal ageing studies (SLAS). J Am Med Dir Assoc. 2017;18:252-8. https://doi.org/10.1016/j. jamda.2016.09.015.

9. Boyle PA, Buchman AS, Wilson RS, Leurgans SE, Bennett DA. Physical Frailty is associated with incident mild Cognitive impairment in community-based older persons. J Am Geriatr Soc. 2010;58:248-55. https://doi.org/10.1111/j. 1532-5415.2009.02671.x.

10. Mitnitski A, Fallah N, Rockwood MR, Rockwood K. Transitions in cognitive status in relation to frailty in older adults: a comparison of three frailty measures. J Nutr Heal Aging. 2011;15:863-7. https://doi.org/10.1007/s12603011-0066-9. 
11. Lee Y, Kim J, Chon D, Lee K-E, Kim J-H, Myeong S, et al. The effects of frailty and cognitive impairment on 3-year mortality in older adults. Maturitas. 2018;107:50-5. https://doi.org/10.1016/j.maturitas.2017.10.006.

12. Fougère B, Delrieu J, del Campo N, Soriano G, Sourdet S, Vellas B. Cognitive Frailty. Mechanisms, tools to measure, Prevention and Controversy. Clin Geriatr Med. 2017;33:339-55. https://doi.org/10.1016/j.cger.2017.03.001

13. Kelaiditi E, Cesari M, Canevelli M, Abellan Van Kan G, Ousset PJ, GilletteGuyonnet S, et al. Cognitive frailty: rational and definition from an (I.A.N.A./I. A.G.G.) international consensus group. J Nutr Heal Aging. 2013;17:726-34. https://doi.org/10.1007/s12603-013-0367-2

14. Zhang Z-W, Cheng J, Xu F, Chen Y-E, Du J-B, Yuan M, et al. Red blood cell extrudes nucleus and mitochondria against oxidative stress. IUBMB Life. 2011;63:560-5. https://doi.org/10.1002/iub.490.

15. Liu Z, Han L, Gahbauer EA, Allore HG, Gill TM. Joint trajectories of cognition and Frailty and associated burden of patient-reported outcomes. J Am Med Dir Assoc. 2018;19:304-309.e2. https://doi.org/10.1016/j.jamda.2017.10.010.

16. Ma L, Zhang L, Zhang Y, Li Y, Tang Z, Chan P, et al. Cognitive Frailty in China: results from China comprehensive geriatric assessment study. Front Med. 2017:4:174. https://doi.org/10.3389/fmed.2017.00174.

17. Ma L, Li Z, Tang Z, Sun F, Diao L, Li J, et al. Prevalence and sociodemographic characteristics of disability in older adults in China: findings from China comprehensive geriatric assessment study. Arch Gerontol Geriatr. 2017;73:199-203. https://doi.org/10.1016/j.archger.2017.07.029.

18. Ma L, Tang Z, Zhang L, Sun F, Li Y, Chan P. Prevalence of Frailty and associated factors in the community-dwelling population of China. J Am Geriatr Soc. 2018;66:559-64. https://doi.org/10.1111/jgs.15214.

19. Li H, Jia J, Yang Z. Mini-mental state examination in elderly Chinese: a population-based normative study. J Alzheimers Dis. 2016;53:487-96. https://doi.org/10.3233/JAD-160119.

20. Han R, Tang Z, Ma L. Related factors of cognitive impairment in communitydwelling older adults in Beijing longitudinal study of aging. Aging Clin Exp Res. 2018. https://doi.org/10.1007/s40520-018-0943-8.

21. Ma L, Han R, Li L, Li Z, Sun F, Diao L, et al. Trends in the prevalence of antihypertensive drug treatment in the Beijing longitudinal study of aging. Arch Gerontol Geriatr. 2018;74:44-8. https://doi.org/10.1016/j.archger.2017.09.004.

22. Shimada H, Makizako H, Tsutsumimoto K, Doi T, Lee S, Suzuki T. Cognitive Frailty and Incidence of Dementia in Older Persons. J Prev Alzheimer's Dis. 2018:5:42-8. https://doi.org/10.14283/jpad.2017.29.

23. Roppolo M, Mulasso A, Rabaglietti E. Cognitive frailty in italian communitydwelling older adults: prevalence rate and its association with disability. J Nutr Heal Aging. 2016;21:631-6. https://doi.org/10.1007/s12603-016-0828-5.

24. Sugimoto T, Sakurai T, Ono R, Kimura A, Saji N, Niida S, et al. Epidemiological and clinical significance of cognitive frailty: a mini review. Ageing Res Rev. 2018;44:1-7. https://doi.org/10.1016/j.arr.2018.03.002.

25. Yu R, Morley JE, Kwok T, Leung J, Cheung O, Woo J. The effects of combinations of Cognitive impairment and pre-frailty on adverse outcomes from a prospective community-based cohort study of older Chinese people. Front Med. 2018;5:50. https://doi.org/10.3389/fmed.2018.00050.

26. Amanzio M, Palermo S, Zucca M, Rosato R, Rubino E, Leotta D, et al. Neuropsychological correlates of pre-Frailty in neurocognitive disorders: a possible role for metacognitive dysfunction and mood changes. Front Med. 2017;4:199. https://doi.org/10.3389/fmed.2017.00199.

27. St. John PD, Tyas SL, Griffith LE, Menec V. The cumulative effect of frailty and cognition on mortality - results of a prospective cohort study. Int Psychogeriatrics. 2017;29:535-43. https://doi.org/10.1017/ S1041610216002088.

28. Tarazona-Santabalbina FJ, Gómez-Cabrera MC, Pérez-Ros P, Martínez-Arnau FM, Cabo H, Tsaparas K, et al. A multicomponent exercise intervention that reverses Frailty and improves cognition, emotion, and social networking in the community-dwelling frail elderly: a randomized clinical trial. J Am Med Dir Assoc. 2016;17:426-33. https://doi.org/10.1016/j.jamda.2016.01.019.

29. Sargent L, Brown R. Assessing the current state of cognitive frailty: measurement properties. J Nutr Health Aging. 2017;21:152-60. https://doi. org/10.1007/s12603-016-0735-9.

30. Liu T, Wong GH, Luo H, Tang JY, Xu J, Choy JC, et al. Everyday cognitive functioning and global cognitive performance are differentially associated with physical frailty and chronological age in older Chinese men and women. Aging Ment Health. 2017:1-6. https://doi.org/10.1080/13607863. 2017.1320700
31. Gale CR, Ritchie SJ, Cooper C, Starr JM, Deary IJ. Cognitive ability in late life and onset of physical Frailty: the Lothian birth cohort 1936. J Am Geriatr Soc. 2017;65:1289-95. https://doi.org/10.1111/jgs.14787.

32. Fougère $B$, Daumas $M$, Lilamand $M$, Sourdet $S$, Delrieu J, Vellas $B$, et al. Association between Frailty and Cognitive impairment: cross-sectional data from Toulouse Frailty day hospital. J Am Med Dir Assoc. 2017;18:990.e1-5. https://doi.org/10.1016/j.jamda.2017.06.024.

33. Godin J, Armstrong JJ, Rockwood K, Andrew MK. Dynamics of Frailty and cognition after age 50: why it matters that Cognitive decline is mostly seen in old age. J Alzheimers Dis. 2017;58:231-42. https://doi.org/10.3233/JAD161280.

34. Bunce D, Batterham PJ, Mackinnon AJ. Long-term associations between physical Frailty and performance in specific Cognitive domains. Journals Gerontol Ser B Psychol Sci. Soc Sci. 2017. https://doi.org/10.1093/geronb/gbx177.

35. Lee JSW, Auyeung T-W, Leung J, Kwok T, Woo J. Transitions in Frailty states among community-living older adults and their associated factors. J Am Med Dir Assoc. 2014;15:281-6. https://doi.org/10.1016/j.jamda.2013.12.002.

36. Umegaki H, Makino T, Shimada H, Hayashi T, Wu Cheng X, Kuzuya M. Cognitive dysfunction in Urban-Community dwelling Prefrail older subjects. J Nutr Health Aging. 2018. https://doi.org/10.1007/s12603-018-1017-5.

37. Ngandu T, Lehtisalo J, Solomon A, Levälahti E, Ahtiluoto S, Antikainen R, et al. A 2 year multidomain intervention of diet, exercise, cognitive training, and vascular risk monitoring versus control to prevent cognitive decline in at-risk elderly people (FINGER): a randomised controlled trial. Lancet. 2015; 385:2255-63. https://doi.org/10.1016/S0140-6736(15)60461-5.

38. Cezar N, Izbicki R, Cardoso D, Almeida J, Valiengo L, Camargo M, et al. Frailty in older adults with amnestic mild cognitive impairment as a result of Alzheimer's disease: a comparison of two models of frailty characterization. Geriatr Gerontol Int. 2017. https://doi.org/10.1111/ggi. 13028.

39. Vella Azzopardi R, Beyer I, Vermeiren S, Petrovic M, Van Den Noortgate N, Bautmans I, et al. Increasing use of cognitive measures in the operational definition of frailty — a systematic review. Ageing Res Rev. 2018;43:10-6. https://doi.org/10.1016/j.arr.2018.01.003.

\section{Ready to submit your research? Choose BMC and benefit from:}

- fast, convenient online submission

- thorough peer review by experienced researchers in your field

- rapid publication on acceptance

- support for research data, including large and complex data types

- gold Open Access which fosters wider collaboration and increased citations

- maximum visibility for your research: over $100 \mathrm{M}$ website views per year

At $\mathrm{BMC}$, research is always in progress.

Learn more biomedcentral.com/submissions 